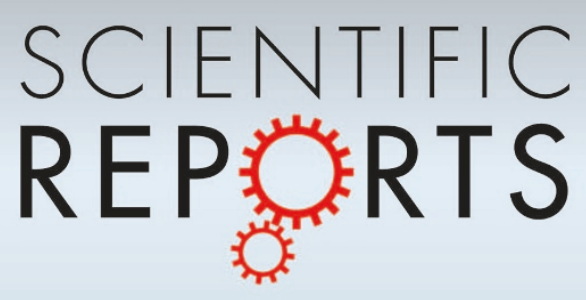

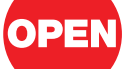

SUBJECT AREAS:

TOPOLOGICAL

INSULATORS

ELECTRONIC PROPERTIES AND

DEVICES

THEORETICAL PHYSICS

ELECTRONIC PROPERTIES AND MATERIALS

Received

20 November 2012

Accepted

12 March 2013

Published

9 April 2013

Correspondence and requests for materials should be addressed to

S.M.A. (savdoshe@ purdue.edu)

\section{Topological Signatures in the Electronic Structure of Graphene Spirals}

\author{
Stas M. Avdoshenko', Pekka Koskinen², Haldun Sevinçli3 ${ }^{3,4,6}$, Alexey A. Popov ${ }^{5}$ \& Claudia G. Rocha ${ }^{2}$
}

\begin{abstract}
${ }^{1}$ School of Materials Engineering, Purdue University, West Lafayette, Indiana, USA, ${ }^{2}$ Nanoscience Center, Department of Physics, University of Jyväskylä, 40014 Jyväskylä, Finland, ${ }^{3}$ DTU Nanotech, Department of Micro- and Nanotechnology, Technical University of Denmark, DK-2800, Denmark, ${ }^{4}$ Institute for Materials Science and Max Bergmann Center of Biomaterials, Dresden University of Technology, 01062 Dresden, Germany, ${ }^{5}$ The Leibniz Institute for Solid State and Materials Research in Dresden, 01069 Dresden, ${ }^{6}$ Center for Nanostructured Graphene, Technical University of Denmark, DK-2800 Kgs. Lyngby, Denmark.
\end{abstract}

Topology is familiar mostly from mathematics, but also natural sciences have found its concepts useful. Those concepts have been used to explain several natural phenomena in biology and physics, and they are particularly relevant for the electronic structure description of topological insulators and graphene systems. Here, we introduce topologically distinct graphene forms - graphene spirals - and employ density-functional theory to investigate their geometric and electronic properties. We found that the spiral topology gives rise to an intrinsic Rashba spin-orbit splitting. Through a Hamiltonian constrained by space curvature, graphene spirals have topologically protected states due to time-reversal symmetry. In addition, we argue that the synthesis of such graphene spirals is feasible and can be achieved through advanced bottom-up experimental routes that we indicate in this work.

$\mathrm{n}$ mathematics, topology analyzes how the properties of objects preserve under continuous deformations. But the interest to topological analysis is not restricted to mathematics alone; it spans also through biology, chemistry and materials science. In protein systems topology determines when protein folding sustains the rest of their cellular life ${ }^{1}$. In condensed matter physics, topology dominates several quantum phenomena, such as quantum-Hall ${ }^{2}$, spin-Hall ${ }^{3}$, and Aharonov-Bohm ${ }^{4}$ effects, as well as the physics of topological insulators ${ }^{5}$.

In topological insulators, the surface electronic states are governed by topological features, making their quantum information robust against impurity scattering. Such robustness, by being protected by timereversal-invariant Hamiltonian, could pave a reliable avenue toward fault-tolerant quantum-computing technology ${ }^{6}$. Experiments via angle-resolved photoemission spectroscopy performed in $\mathrm{Bi}_{2} \mathrm{Se}_{3}$ compounds ${ }^{7}$ and $\mathrm{Bi}_{1-x} \mathrm{Sb}_{x}$ alloys ${ }^{8}$ have shown signatures specific to topological insulators, such as large bulk energy gap and a single-surface Dirac cone associated to its topologically protected state. Dirac cones make the physics of graphene and topological insulators similar, even though graphene has two Dirac valleys with spin degeneracy while topological insulators have only one Dirac valley without spin degeneracy ${ }^{9}$. In addition, graphene can exhibit topologically protected quantum-Hall states with applied perpendicular and periodic magnetic fields ${ }^{10}$.

In the absence of structure inversion symmetry, surface states may split because of Rashba spin-orbit interaction ${ }^{11}$. This splitting has been verified in thin films ${ }^{12}$ and semiconductor heterostructures having an inversion asymmetry of the confining potentials ${ }^{13-15}$. The splitting arises from the Rashba interaction Hamiltonian, $H_{R}=\alpha(\vec{E} \times \vec{p}) \cdot \vec{s}$, where $\alpha$ is the Rashba coefficient, $\vec{s}$ is the spin of an electron moving with momentum $\vec{p}=\hbar \vec{k}$ in an electric field $\vec{E}^{16}$. In a two-dimensional non-interacting electron gas, therefore, Rashba spin-orbit interaction splits the parabolic energy bands in two, $\epsilon_{ \pm}(k)=\hbar^{2} k^{2} / 2 m^{*} \pm \alpha k$, where $m^{*}$ is the effective mass ${ }^{17}$. Even though spin-orbit interactions are often intrinsic, such as the spin-orbit-induced $\sim 0.1 \mathrm{meV}$ energy gap in graphene ${ }^{18}$, Rashba splitting is interesting for applications because the control over an external electric field makes it extrinsic and tunable ${ }^{19}$.

Extrinsic spin-orbit manipulation in graphene has been probed by external electric fields ${ }^{20}$, by doping ${ }^{21}$, by mechanical folding ${ }^{22}$ and by depositing graphene on substrates ${ }^{23}$. Especially substrate interfaces, by always involving inversion asymmetries, strengthen Rashba interaction ${ }^{24}$. In graphene-based structures, however, the origin of the Rashba interaction is unlike in any other material: the interaction, although similar to the usual spinorbit effects, arises not from the real electron spin, but from the spin related to the two non-equivalent atomic sites in the unit cell, the pseudospin. In graphene the Rashba splitting occurs around $k$-points displaying time-reversal symmetry ${ }^{25}$, meaning that the splitting is seen around Brillouin zone centers and zone boundaries ${ }^{26}$. In graphene the zone boundary points $K$ and $K^{\prime}$ are non-equivalent, but they have the same energy and they are connected by 
time-reversal symmetry ${ }^{27-29}$. This symmetry makes the connection to topological insulators. The symmetry protects a pair of gapless helical edge modes in topological insulators belonging to $\mathrm{Z}_{2}$ class $^{30}$. In a prototype of such topological insulator, an intrinsic spinorbit coupling induces a topological mass term in the electronic structure of an atomic hexagonal frame $\mathrm{e}^{31,32}$. Also, it is important to mention that most often $\mathrm{Z}_{2}$ class materials are discussed, at least experimentally, including heavy elements with strong spin-orbital coupling like $\mathrm{Bi}_{2}(\mathrm{Se}, \mathrm{Te})_{3}$ compounds ${ }^{7}$.

Till now spin-orbit effects with highly protected topological edgestates in graphene have been obtained only by external perturbations such as external fields, heavy mechanical distortions, or chemical doping. In this letter, therefore, we investigate the above-discussed topological signatures in distinct graphene systems: the graphene spirals (see Fig. 1). As a central result, we find out that the spiral topology creates an intrinsic Rashba splitting. While in canonical illustrations of the effect in which an external magnetic field is applied to induce electron precession, in graphene spirals the track in which electrons move already displays an helical topology; electrons are constrained to move along an helical path in k-space and, due to unbreakable structure inversion symmetry, the Rashba-like band structure topology becomes an intrinsic effect for this material class. Our results demonstrate that graphene spirals naturally possess robust topological states as those observed in topological insulators.

\section{Results}

Graphene spirals are distinct from the helical graphene motifs reported earlier, such as graphene stripes and ribbons bent to spiral-like shapes ${ }^{33-36}$. In those earlier motifs, the starting point has been a graphene ribbon itself, with regular edges and curvature- or strain-modified $\pi$-electron system ${ }^{37,38}$. In such systems there has always been one-to-one mapping between the helical structure and flat graphene. In other words, it is always possible to build such systems by cutting them out of an infinite and flat graphene sheet. On the contrary, in graphene spirals such a mapping does not exist (see Fig. 1). Spirals are one-dimensional systems, while they still have a graphite layered structure containing perfect hexagons. Edge profiles alternate between armchair and zigzag shapes. Since spirals' local structures resemble graphene, they facilitate chiral topology without overly perturbing the $\pi$-electron system. The largest (a)

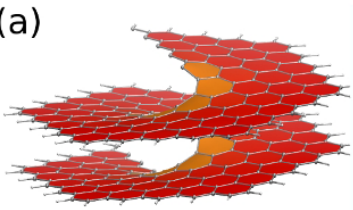

(b)

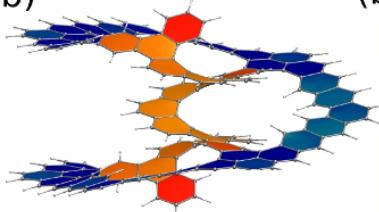

(c)

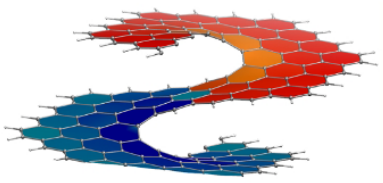

$\left(a^{\prime}\right)$

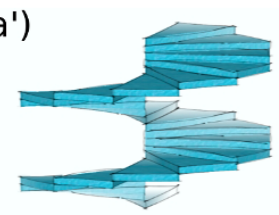

(b')

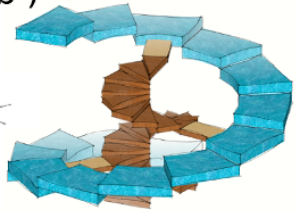

(c')

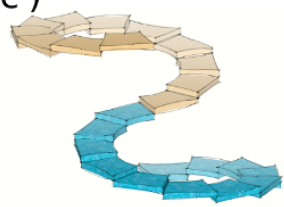

Figure 1 | Examples of graphene spirals. (a) Right-handed spiral, (b) interconnected right- and left-handed double spiral, and (c) loxodrome-like spiral. (a-c) Mapping of the (a-c) structures into helical stairs to highlight the topology of their curved space. perturbations take place at the inner edge of spirals where the strain is largest.

First, we illustrate a notation scheme that we elaborated to identify the graphene spiral models (see Fig. 2). This notation is based on the number of armchair (ac) and zigzag (zz) segments used to complete a full coil of the spiral along the inner and outer edges. We established that the spiral axis goes through the center of a hexagon where $\pi / 6$-symmetry lines merge. Distinct spiral classes can be formed by combining ac and zz segments. The logic of our notation scheme can be better illustrated by projecting the spiral systems onto the plane. According to our construction rules, the spirals can only be formed by hexagons, their edges can simply contain ac- and zz-fragments and there must be no untangled bonds. How wide is the spiral can be determined by the outlined circles with radii $r_{1}, r_{2}, \ldots, r j$ being $r_{j}=$ $1.23+2.46 j \AA$. These circles always enclose the first series of ac segments crossing the symmetry line $\mathrm{S} 1$. The remaining of the spiral is completed by as much $\mathrm{zz}$ segments required to maintain the perfect hexagonal frame. In this way, we define the notation $\operatorname{ac}[m] \mathrm{zz}\left[n_{\text {in }}-n_{\text {out }}\right]$, where $m$ is the number of ac units and $n_{\text {in/out }}$ is the number of $\mathrm{zz}$ units used to complete, respectively, the spiral inner and outer edges. Spirals with ac segments which are flipped towards the origin have an additional "*” in their notation, e.g. ac*[2]zz[1-3] [see Fig. 2 (c) and (d)]. These conventions impose the spirals to be invariant under an axial translation of $b$ (interlayer separation), under an axial translation of $b / 6$ combined with a rotation of $\pi / 3$. Furthermore, it is worth mentioning that almost any parametric curve can be used to represent edges formed under such hexagonal basis, however analysis involving more complex edge topologies are far beyond the scope of this work. The geometry and electronic structure of the built graphene spirals were investigated within density functional theory (DFT) implemented within SIESTA package ${ }^{39,40}$. To confirm our results, SIESTA calculations were compared to other methods such as single $\pi$-band tight-binding (see Supplementary material), density-functional tight-binding implemented within DFTB + code $^{41,42}$, and VASP density-functional package $^{43}$. Detailed description of the used parameters and calculation (a)

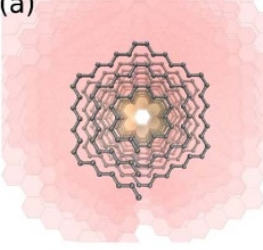

(d)

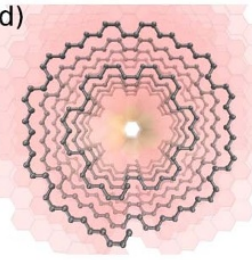

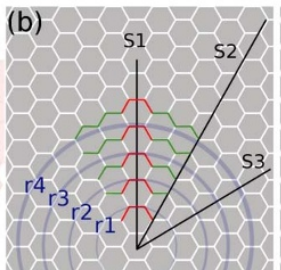

(e)

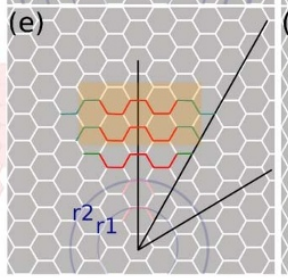

(c)

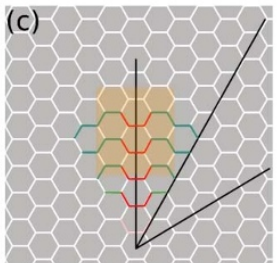

(f)
Figure 2 Notation of graphene spirals edges: Right, Middle and left panels depict three-dimensional (3D) top view, conventional, and mirrored spirals with flipped ac segments, respectively. Note that on the right panels only carbon-carbon bonds along the inner and outer edges of the spirals are highlighted. Armchair (ac) segments are marked in red while zigzag (zz) segments are drawn in green colour. S1, S2 and S3 black lines are the hexagonal symmetry axes ( $\pi / 6$ wedge angles) and pass through the origin point located on the center of the spiral. The blue circles outline possible extensions that the spirals can hold along their inner and outer edges. (a) 3D view of ac [1]zz[0-4], with its planar projection shown in (b). (c) Planar projection of ac*[1]zz[0-4] spiral where ac segments are flipped. (d) 3D view of ac*[2]zz[1-3], with its planar projection shown in (f). (e) Planar projection of ac[2]zz[1-3] spiral. 
conditions are presented in the end of the manuscript. We obtained the band structures for distinct graphene spiral models which had their atomic configuration fully optimized.

$A b$ initio band structures obtained for graphene spirals of different widths are shown in Fig. 3. One can see that the two narrower spirals are semiconductors with an energy gap of approximately 2.3 and $1.0 \mathrm{eV}$ for $\mathrm{ac}[1] \mathrm{zz}[0]$ and $\mathrm{ac}[1] \mathrm{zz}[0-1]$, respectively. Differently, $\mathrm{ac}[1] z z[0-2]$ and $\mathrm{ac}[1] z z[0-3]$ reveal semimetallic states. However, more intriguing than the change of electronic character in response to geometrical variations is the peculiar band splitting at the Brillouin zone center. These band splittings represent the Rashba effect, the topological signatures in the electronic structure of graphene spirals. This is our central result. The splitting is robust and present in all spirals. The origin for the Rashba effect is related to how the Dirac particles in graphene couple with the spiral curved space. Graphite crystals, for instance, already manifest a natural splitting of the $\pi$ bands close to the Fermi level due to the two non-equivalent carbon atoms in the unit cell. Since the number of atoms in the spiral unit cell - assumed to be an unique coil - is considerably higher, one expects a superior number of splittings in these systems. In addition, the interlayer interaction in spirals also plays an important role. The usual graphite layer stacking provides an uniform potential profile over the whole sample which is not the case for the spirals. They possess chiral symmetry which leads to electron-hole symmetry breaking. Under chiral symmetry operation $\hat{S}_{\theta}^{\tau}$, the wavefunction composed of a set of molecular orbitals within the unit cell get translated (by $\tau$ ) and rotated (by $\theta$ ) simultaneously ${ }^{44}$. The operator $\hat{S}_{\theta}^{\tau}$ replaces the translation operator $\hat{T}$ in Bloch's theorem ${ }^{45}$. It is therefore important to distinguish reciprocal $\mathrm{k}$-vectors of the linear $(k)$ and curved or chiral $\left(k^{\prime}\right)$ systems. These vectors are related by $k^{\prime}=$ $(b / \tau) k$, as can be demonstrated by applying $\hat{S}$ consecutively until a full

(a)

(b)
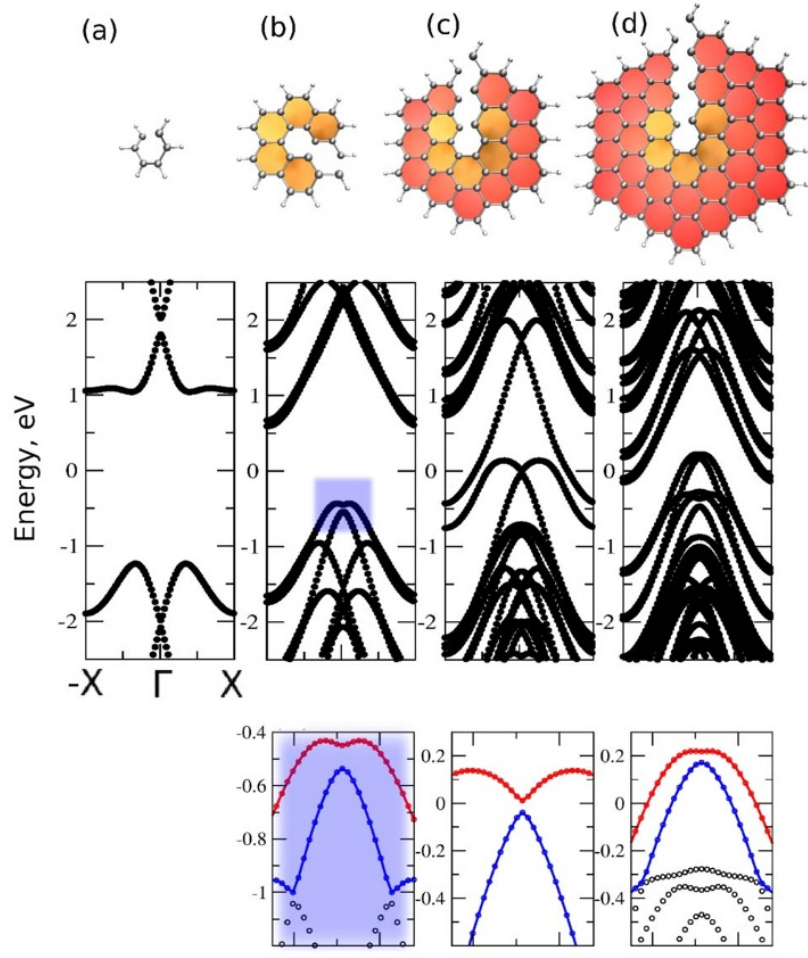

Figure 3 Band structures of graphene spirals. (a) ac $[1] \mathrm{zz}[0]$,

(b) ac[1]zz[0-1], (c) ac[1]zz[0-2], and (d) ac[1] zz[0-3]. Fermi energy is set at $0 \mathrm{eV}$. Vertical axes span the $k$-vector intervals $[-\pi / b, \pi / b]$. Narrow spirals, semiconductors with $2.3-1.0 \mathrm{eV}$ energy gaps; wide spirals, semimetals. Bottom panels depict a zoom over low energy bands around the $\Gamma$. The band closest to the Fermi energy is highlighted in red while the second closest is colored in blue. turn is completed. The enlarged Brillouin zone in chiral systems is often referred to the Jones zone $(\mathrm{JZ})^{46}$. It can be shown that in a constantly curved space systems, a bisector reduction of whole the Brillouin space must have two components as for any system described by a Hamiltonian under chiral symmetry.

Let us now illustrate in detail the origin of the Rashba effect in our graphene spirals by considering a linear chain model as starting point (see also Supplementary material). The chain periodicity is given by the unit cell length $a$, and chain's one-dimensional potential obeys $V(z)=V(z+a)$. When the unit cell gets more atoms, the reciprocal space shrinks, the bands fold and their number increases. For unit cells with an even number of atoms, two types of bands exist: bands crossing the Fermi level (i) at the $\Gamma$ point and (ii) at the edges of the Brillouin zone. At the $\Gamma$ point, the energy states are quantized as for a finite ring with $M$ atoms, $\epsilon_{n}(k)=-2 t \cos (2 \pi n / M)$, where $n=1, \ldots$, $M$ and $t$ is the hopping parameter. Only when $2 n / M= \pm 1 / 2, \pm 3 /$ $2, \pm 5 / 2, \ldots$, a band will cross the Fermi level at $\Gamma$ point. For unit cells with an odd number of atoms, the band crossing is shifted from $\Gamma$ point and an energy gap opens. A reminiscence of this cosine trend exists also in polyacetylene-like spiral (not shown). For spirals with large circumference arcs, the low-energy band slopes at the edges of the JZ are markedly higher, meaning curvature-dependent Fermivelocities. This dependence is absent in single- $\pi$ band tight-binding model since it cannot account for curvature effects. Furthermore, in these spirals the chiral operation changes the orbital orientation. The angular parts of the p-orbitals can be written as $p_{x}(l)=p_{x} \cos (l \theta)$, $p_{y}(l)=p_{y} \sin (l \theta)$, and $p_{z}(l)=p_{z}$, where $l$ is an integer number for consecutive units cells of two non-equivalent carbon atoms. This orbital orientation changes the phase of the wavefunction, a $180^{\circ}$ turn flips its sign; this property modifies the profiles of bonding and antibonding bands markedly, due to additional cross-coupling terms ${ }^{46,47}$. In the simpler spiral example shown on Fig. 3(a), its valence band develops minima at $\mathrm{X}$-points and maxima around the $\Gamma$-point. As the spiral becomes wider, the electronic structure becomes richer but still such peculiar band offset is maintained. This energy uplift and concomitant maximum development around the $\Gamma$ point arises because the states become more antibonding due to chiral symmetry.

Zooming over the low energy bands around $\Gamma$ point [bottom panels of Fig. 3], one can notice that they display intriguing anticrossings close to the Fermi energy as the spirals get wider. This occurs due to certain selection rules (similar to those manifested in ordinary atomic rings) that the helical states must obey combined with the chiral symmetry of the curved space. These anticrossings between such chiral branches can be finely tuned by means of external fields. This is shown on Fig. 4 which depicts the band structure for the spiral ac[1]zz[0-2] while a high intense external electric field is applied perpendicularly its axial direction. At such intense electric fields, one would expect more impacting changes on the electronic structure of the spirals. Nonetheless, such robustness is simply a remarkable proof that these helical modes are protected by time-reversal symmetry. We can allude the nature of these states by assuming that the spirals are composed of $q$ concentric rings embedded in the same unit cell. if the spiral respects hexagonal symmetry, i.e. each ring contains a number of atoms such as $M=6,18,30,42, \ldots$, according to a tight-binding description, whenever $q$ is even, an energy gap opens in the electronic structure (see Fig. 2 in supplementary information). Otherwise, metallic edge-states touching the Brillouin zone boundaries will form. The same opening-closing rules for the energy gap fail for the first principle results since they take into account curvature effects. However the low energy states still retain its edge-nature as can be seen from the isosurface plots of the local density of states at the Fermi energy for the spiral ac[1] zz[0-2] (see Fig. 3 in supplementary material). Ultimately, by comparing the results derived from $a b$ initio and tight-binding methods, we successfully confirmed that the electronic structure of graphene spirals couples to the helical space backbone. 

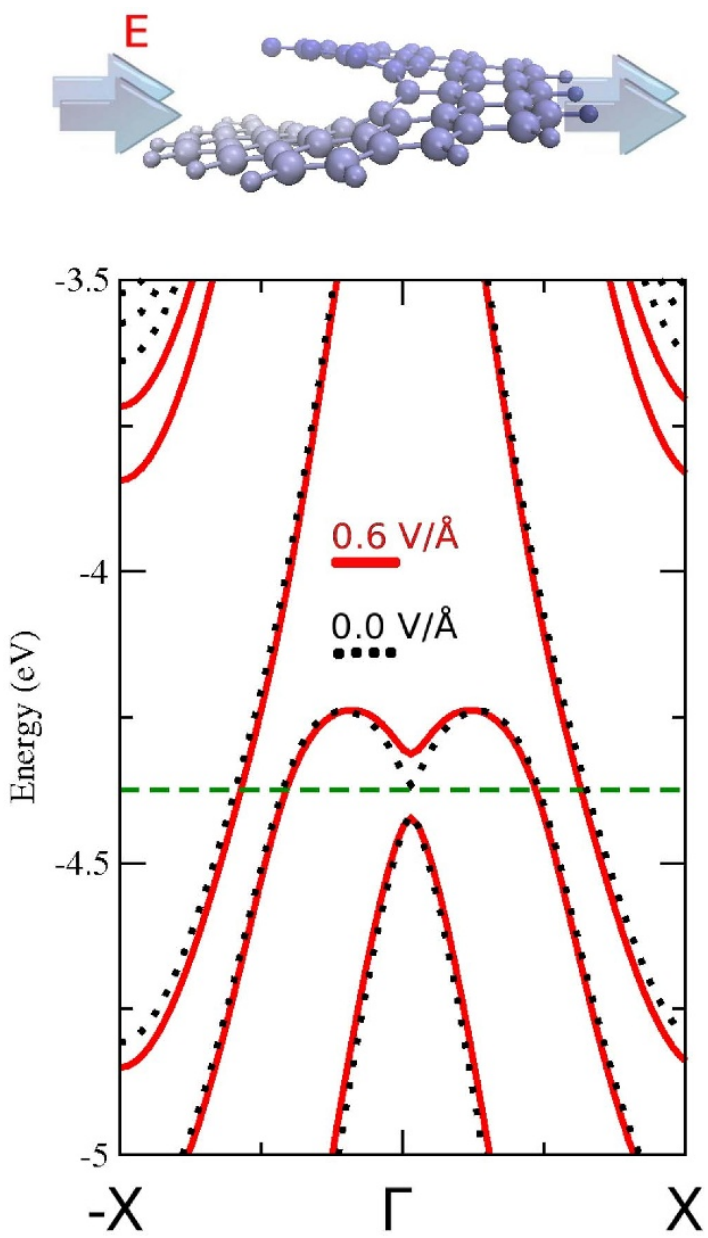

Figure $4 \mid$ (red lines) Band structure for the graphene spiral ac[1]zz[0-2] (top panel) under the influence of an external electric field of intensity $0.6 \mathrm{~V} / \AA$ and applied perpendicularly to its axial direction. Horizontal line marks the Fermi energy (not shifted to zero). Dotted black lines are the energy dispersion for the same spiral for null electric field.

\section{Discussion}

Previous works have reported that ripples in graphene can be modeled by coupling the Dirac equation to a curved metric space defined phenomenologically from corrugations observed in experiments ${ }^{48,49}$. Such covariant formalism gives rise to an effective Hamiltonian where it is possible to identify that the electrons on the deformed space exhibit a new Fermi velocity. The latter is smaller than the flat graphene case. Their findings were confirmed by Raman spectroscopy measurements performed in folded graphene samples ${ }^{50}$. In our spiral examples, electrons propagating along the helical track with stronger bending would then manifest smaller effective Fermi velocities in comparison to those with smoother curvature. This difference can be clearly seen on the band structures of 3 where the low energy bands associated to each spiral have distinct slopes. Such velocity reduction cannot be captured by the single $\pi$-band tight binding approximation since curvature is invisible within this description. Another prominent feature that can be distinguished from the effective covariant Hamiltonian is the appearance of an effective magnetic field pointing perpendicularly to the deformed graphene sheet ${ }^{49,51}$. In other words, mechanical strain loaded on the distorted section of the graphene mimics the same effects caused by an external magnetic field (see supplementary information where we demonstrate how the Rashba-like Hamiltonian can be derived from an illustrative toy-model example). The field intensity is strictly related to how sharp the curvature is. This effective field appears naturally from covariant formalism where Dirac equation is solved on curved space. Once the metric of the deformed space is known, this information is plugged on the Dirac equation and techniques resembling to perturbation theory can be used if one assumes that the curvature is rather smooth. The solution for this problem can be often recognized as the standard graphene Dirac model in the presence of an effective potential generated by its own curvature. Such method is very efficient in dealing with smooth ripples or light corrugations on the graphene sheet. Extending such interpretation to our systems, one can already expect that the highly intensified curvature of the graphene spirals will affect enormously their electronic response. Since the curvature of the graphene spirals is so remarkable, the use of covariant methods following expansion procedures is unreliable. In this sense, we must indeed rely on robust ab initio methods where all the structures are fully optimized and the effects of the curvature are naturally incorporated in the Hamiltonian.

But even if unreliable, the formalism can help understand the origin of the Rashba splitting. In Aharonov-Bohm (AB) devices real magnetic fields induce electron wavefunctions an additional phase due to the breaking of time-reversal. The phase is proportional to magnetic flux penetrating the device geometry and depends on whether electron moves clockwise or anticlockwise ${ }^{52}$. For the sake of simplicity, let us consider a spiral chain with 18 atoms in its unit cell $\left(\mathrm{C}_{18}\right)$ and investigate its density of states while varying the unit cell length or the curvature of its helix [see Fig. 5(b)]. This result was obtained within density functional tight-binding (DFTB) formalism in which the atomic structure of the system-chain could be fully optimized for each unit cell length. Electrons moving clockwise $(k+)$ and anticlockwise $(k-)$ acquire different phase factors in their wavefunctions depending on the local curvature of the spiral. Thus, an oscillatory pattern akin to $\mathrm{AB}$ interference emerges in the density of states [see Fig. 5 (a)]. Only here the AB oscillations arise not from a real magnetic field but from a pseudo-magnetic field defined by the system topology; the curvature can be seen as a parameter replacing a real external magnetic field to produce the same effect. As a result, electrons having different pseudospin and being coupled to the pseudo-magnetic field can move in opposite directions and still preserve time-reversal symmetry while exhibiting non-zero $\mathrm{AB}$ phase.

Because of electronic properties similar to topological insulators, graphene spirals could naturally be used in quantum computing.
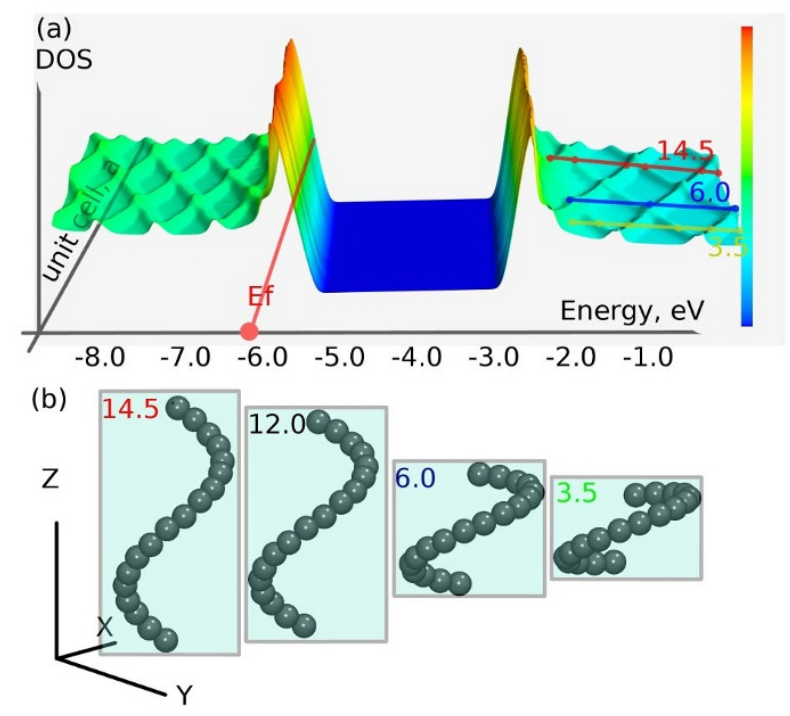

Figure 5 | (a) Aharonov-Bohm-like oscillations in elongated $\mathrm{C}_{18}$ atomic chain being gradually deformed into a spiral shape. Normalized density of states $v s$. energy and elongation of the unit cell (in Angstroms). Line at $E_{f}$, Fermi-level. Horizontal lines highlight the results for some chain geometries that are displayed on panel (b). 
Furthermore, the spiral geometry itself suggests usage as electronics components, as nano-solenoids to produce local magnetic fields. We can estimate the magnetic field intensity, $B$, created by electrons traveling around the spiral of radius $R$. By setting the Lorentz force and the centripetal force equal, we obtain $B=m v_{F} / e R$, where $m$ is the electron mass, $e$ the electron charge, and $v_{F}$ the Fermi velocity. Assuming $R \approx 1 \mathrm{~nm}$ and $v_{F} \approx 10^{6} \mathrm{~m} / \mathrm{s}$ (Fermi-velocity of flat graphene) we obtain an estimate for graphene spiral -generated magnetic field as $B=10^{3} \mathrm{~T}$. The estimate depends on the Fermi-velocity, which our results shows to depend on the precise atomic structure of the spiral. The spiral structure can hence be used to sensitively customize the solenoid properties.

Finally, we discuss the feasibility to realize spirals experimentally. We note that natural growth often favors chiral molecules, first and most notable example being the DNA. Second example is carbon nanotubes grown into helical conformations by using controlled creation of pentagon and heptagon defects during the growth process $^{53,54}$. To synthesize graphene spirals, however, the most relevant experiment was the recent controlled synthesis of narrow graphene nanoribbons ${ }^{55}$. Ribbons were synthesized for controlled ribbon widths, controlled edge shapes and -in particular - controlled topologies. The control was achieved by organic chemical reactions on gold surfaces, where the topology of the nanoribbon was determined by the topology of the precursor monomers. Here, we propose that graphene spirals could be synthesized by the same controlled bottom-up approach. One would only need to choose appropriate precursor monomers, preferably from an organic polymer family with a helical motif. There are also other synthesizing alternatives. Namely, the graphene spiral ac[1] zz[0-1] is already a familiar molecule, the helicene ${ }^{56}$. Fairly long helicenes have been synthesized, and perhaps related techniques could be extended to synthesize also wider graphene spirals ${ }^{33,57}$. Yet another alternative to spiral synthesis is to use the viewpoint of array of screw dislocations ${ }^{54,58}$. It was reported recently, that in nature closely related structures can be found as shown by Rakovan \& Jaszczak ${ }^{59}$.

To conclude, the electronic structure of graphene spirals show Rashba splitting as a distinct topological signature. The splitting can be understood as a consequence of the intrinsic curvature present in graphene spirals, as a consequence of the coupling between pseudo-spin and the curved helical geometry. The splitting mechanism is similar to the mechanism of band inversion in topological insulators ${ }^{60,61}$. The split, low-energy states around $\Gamma$-point are localized at the edges and are protected by the spiral topology, being thus robust against impurities or lattice distortions. These unique electronic properties require neither an external magnetic field nor spinorbit interaction, which is unlike any typical quantum Hall system. Therefore, graphene spirals ought to deserve a prominent role as a fundamental graphene topology, comparable to the topologies of carbon nanotubes and graphene nanoribbons.

\section{Methods}

Presented band structure calculations were performed using the SIESTA ${ }^{39,40}$ package within generalized gradient approximation for the exchange and correlation energies ${ }^{62,63}$. Norm-conserving pseudopotentials ${ }^{64}$ with relativistic corrections and a split-valence double- $\zeta$ basis of pseudoatomic orbitals with an orbital confining energy of $0.05 \mathrm{eV}$ and an energy cutoff of $150 \mathrm{Ry}$ were used, with Perdew-Burke-Ernzerhof functional ${ }^{62,63}$. The k-point sampling contains $6 \mathrm{k}$-points along the spiral axis (simulation cell has length $b$ along spiral axis). Spirals were optimized using Hellmann-Feynman forces down to $0.01 \mathrm{eV} / \AA$ tolerance ${ }^{65,66}$. Because of an elastic axial stress, the relaxation resulted in an average layer separation of $b \approx 3.2 \AA$, somewhat smaller than graphite interlayer distance. Therefore, because van der Waals forces are much weaker than elastic forces, and because their role for the electronic structure is insignificant, they could be safely neglected. Also discussion in this work weakly might be affected by the fact that super cell optimization shows a possible for smallest spiral to change a hexagonal base to a pentagonal. For VASP calculations, the projector augmented wave and generalized gradient approximation for exchange and correlation energy were used ${ }^{62,63}$. Kohn-Sham orbitals were expanded in plane-wave basis set with energies up to $550 \mathrm{eV}$ and the Brillouin zone was sampled over $1 \times 1 \times 4$ Monkhorst-Pack grid. For the pseudopotentials,
Vanderbilt's ultrasoft potentials ${ }^{67}$ with cutoff energy of 58 Ry was used. All used first-principle methods gave results in excellent agreement.

1. Von Heijne, G. Membrane-protein topology. Nature Reviews 7, 909 (2006).

2. Avron, J. E., Osadchy, D. \& Seiler, R. A Topological look at Quantum Hall effect. Physics Today, 38, August 2003.

3. Bernevig, B. A., Hughes, T. L. \& Zhang, S. C. Quantum Spin Hall effect and Topological Phase Transition in HgTe Quantum Wells. Science 314, 1757 (2006).

4. Kobe, D. H. Berry phase, Aharonov-Bohm effect and topology. Journal of Physics A: Mathematical and General 24, 3551 (1991).

5. Brumfiel, G. Star Material. Nature (News feature) 466, 310 (2010).

6. Moore, J. Topological insulators: the next generation. Nature Physics 5, 378 (2009).

7. Pan, Z.-H. et al. Electronic Structure of the Topological Insulator $\mathrm{Bi}_{2} \mathrm{Se}_{3}$ Using Angle-Resolved Photoemission Spectroscopy: Evidence for a Nearly Full Surface Spin Polarization. Physical Review Letters 106, 257004 (2011).

8. Roushan, P. et al. Topological surface states protected from backscattering by chiral spin texture. Nature 460, 1106 (2009).

9. Dubois, S. M.-M., Zanolli, Z., Declerck, X. \& Charlier, J.-C. Electronic properties and quantum transport in Graphene-based nanostructures. The European Physical Journal B 72 1-24 (2009).

10. Haldane, F. D. M. Model for a Quantum Hall Effect without Landau levels: Condensed-Matter realization of the Parity Anomaly. Physical Review Letters 61, 2015 (1988).

11. Shan, W.-Y., Lu, H.-Z. \& Shen, S.-Q. Effective continuous model for surface states and thin films of three-dimensional topological insulators. New Journal of Physics 12, 043048 (2010).

12. Zhang, Y. et al. Crossover of the three-dimensional topological insulator $\mathrm{Bi}_{2} \mathrm{Se}_{3}$ to the two-dimensional limit. Nature Physics 6, 584 (2010).

13. Krebs, O., Rondi, D., Gentner, J. L., Goldstein, L. \& Voisin, P. Inversion Asymmetry in Heterostructures of Zinc-Blende Semiconductors: Interface and External Potential versus Bulk Effects. Physical Review Letters 80, 5770 (1998).

14. Pfeffer, P. Effect of inversion asymmetry on the conduction subbands in GaAs$\mathrm{Ga}_{1-x} \mathrm{Al}_{x}$ As heterostructures. Physical Review B 5915902 (1999).

15. Lechner, V. et al. Tuning of structure inversion asymmetry by the $\delta$-doping position in (001)-grown GaAs quantum wells. Applied Physics Letters 94, 242109 (2009).

16. Rashba, E. I. Fiz. Tverd. Tela (Leningrad) 2, 1224 (1960).

17. Smirnov, S., Bercioux, D. \& Grifoni, M. Bloch's theory in periodic structures with Rashba's spin-orbit interaction. European Physics Letters 80, 27003 (2007).

18. Min, H. et al. Intrinsic and Rashba spin-orbit interactions in graphene sheets. Physical Review B 74, 165310 (2006).

19. De Carvalho, H. B. et al. Electric-field inversion asymmetry: Rashba and Stark effects for holes in resonant tunneling devices. Physical Review B 74, 041305(R) (2006).

20. Gmitra, M., Konschuh, S., Ertler, C., Ambrosch-Draxl, C. \& Fabian, J. Bandstructure topologies of graphene: Spin-orbit coupling effects from first principles. Physical Review B 80, 235431 (2009).

21. Pi, K. et al. Manipulation of Spin Transport in Graphene by Surface Chemical Doping. Physical Review Letters 104, 187201 (2010).

22. Abdelouahed, S., Ernst, A., Henk, J. \& Mertig, I. Spin-split electronic states in graphene: Effects due to lattice deformation, Rashba effect, and adatoms by first principles. Physical Review B 82, 125424 (2010).

23. Gong, S. J. et al. Spintronic properties of graphene films grown on $\mathrm{Ni}(111)$ substrate. Journal of Applied Physics 110, 043704 (2011).

24. Dedkov, Y. S., Fonin, M., Rudiger, U. \& Laubschat, C. Rashba effect in the graphene/Ni(111) system. Physical Review Letters 100, 107602 (2008).

25. Oguchi, T. \& Shishidou, T. The surface Rashba effect: a k• p perturbation approach. Journal of Physics: Condensed Matter 21, 092001 (2009).

26. Nagano, M., Kodama, A., Shishidou, T. \& Oguchi, T. A first-principles study on the Rashba effect in surface systems. Journal of Physics: Condensed Matter 21, 064239 (2009).

27. Morpurgo, A. F., \& Guinea, F. Intervalley Scattering, long-range disorder, and effective time-reversal symmetry breaking in graphene. Physical Review Letters 97, 196804 (2006).

28. L. Lenz \& Bercioux, D. Dirac-Weyl electrons in a periodic spin-orbit potential EPL 96, 27006 (2011).

29. Ortix, C., Yang, L. \& Van den Brink, J. Graphene on incommensurate substrates: trigonal warping and emerging Dirac cone replicas with halved group velocity. Phys. Rev. 86, 081405 (2012).

30. Imura, K.-I., Mao, S., Yamakage, A. \& Kuramoto, Y. Flat edge modes of graphene and of $\mathrm{Z}_{2}$ topological insulator. Nanoscale Research Letters 6, 358 (2011).

31. Kane, C. L. \& Mele, E. J. Quantum Spin Hall Effect in Graphene. Physical Review Letters 95, 226801 (2005).

32. Kane, C. L. \& Mele, R. J. Z Topological order and the Quantum Spin Hall Effect. Physical Review Letters 95, 146802 (2005).

33. Sehnal, P. et al. An organometallic route to long helicenes, PNAS 106, 13169 (2009).

34. Xu, Z. P. \& Buehler, M. J. Geometry Controls Conformation of Graphene Sheets: Membranes, Ribbons, and Scrolls. ACS Nano, 4, 3869-3876 (2010). 
35. Li, Y., Sun, F. \& Li, H. Helical Wrapping \& Insertion of Graphene Nanoribbon to Single-Walled Carbon Nanotube. The Journal of Physical Chemistry C 38, 18459 (2011).

36. Kit, O. O., Tallinen, T., Mahadevan, L., Timonen, J. \& Koskinen, P. Twisting Graphene Nanoribbons into Carbon Nanotubes. Physical Review B 85, 085428 (2012).

37. Bets, K. V. \& Jacobson, B. I. Spontaneous Twist \& Intrinsic Instabilities of Pristine Graphene Nanoribbons. Nano Research 2, 161 (2009).

38. Koskinen, P. Electromechanics of twisted graphene nanoribbons. Applied Physics Letters 99, 013105 (2011).

39. Artacho, E., Sanchez-Portal, D., Ordejón, P., García, A. \& Soler, J. M. Linearscaling ab-initio calculations for large and complex systems. Phys. Stat. Sol. 215 809 (1999).

40. Soler, J. M. et al. The Siesta method for ab initio order-N materials simulation. J. Phys. Cond. Matter 14, 2745 (2002).

41. Density Functional based Tight Binding (DFTB+, 2012), http://www. dftb-plus.info.

42. Elstner, M. et al. Self-consistent-charge density-functional tight-binding method for simulations of complex materials properties. Physical Review B 58, 7260 (1998).

43. Kresse, G. \& Furthmuller, J. Efficient iterative schemes for ab initio total-energy calculations using a plane-wave basis set. Physical Review B 54, 11169 (1996).

44. Blumen, A. \& Merkel, C. Energy Band Calculations on Helical Systems. Physica Status Solidi (b) 83, 425 (1977)

45. Koskinen, P. \& Kit, O. O. Efficient approach for simulating distorted nanomaterials. Physical Review Letters 105, 106401 (2010)

46. Glassey, W. V. \& Hoffmann, R. Band structure representations of the electronic structure of one-dimensional materials with helical symmetry. Theoretical Chemistry Accounts 107, 272 (2002).

47. Kollmar, C. \& Hoffmann, R. Polyisocyanides: Electronic or Steric Reasons for their Presumed Helical Structure? Journal of the American Chemical Society 112, 8230 (1990).

48. González, J. \& Herrero, J. Graphene wormholes: a condensed matter illustration of Dirac fermions in curved space. Nuclear Physics B 825, 426 (2010).

49. Vozmediano, M. A. H., De Juan, F. \& Cortijo, A. Gauge fields and curvature in graphene. Journal of Physics: Conference Series 129, 012001 (2008).

50. Ni, Z., Wang, Y., Yu, T., You, Y. \& Shen, Z. Reduction of Fermi velocity in folded graphene observed by resonance Raman spectroscopy. Physical Review B 77, 235403 (2008).

51. Huertas-Hernando, D., Guinea, F. \& Brataas, A. Spin-orbit-mediated spin relaxation in graphene. Physical Review Letters 103, 146801 (2009).

52. De Juan, F., Cortijo, A., Vozmediano, M. A. H. \& Cano, A. Aharonov-Bohm interferences from local deformations in graphene. Nature Physics 7, 810 (2011).

53. Gao, R., Wang, Z. L. \& Fan, S. Kinetically Controlled Growth of Helical and Zigzag Shapes of Carbon Nanotubes. The Journal of Physical Chemistry B 104, 1227 (2000).

54. Morin, S. A., Bierman, M. J., Tong, J. \& Jin, S. Mechanism and Kinetics of Spontaneous Nanotube Growth Driven by Dislocations. Science, 328, 476 (2010).

55. Cai, J. et al. Atomically precise bottom-up fabrication of graphene nanoribbons. Nature 466, 470 (2010).

56. Cornelissen, J. J. L. M., Rowan, A. E., Nolte, R. J. M. \& Sommerdijk, N. A. J. M. Chemical Reviews 101, 4039 (2001).
57. Shen, Y. \& Chen, C.-F. Helicenes: Synthesis and Applications. Chemical Reviews 112, 1463 (2012)

58. Paisley, E. A. et al. Surfactant-enabled epitaxy through control of growth mode with chemical boundary conditions. Nature Communications 2, 461 (2011).

59. Rakovan, J. \& Jaszczak, J. A. American Mineralogist 87, 17-24 (2002).

60. Fu, L. \& Kane, C. L. Topological insulators with inversion symmetry. Physical Review B 76, 045302 (2007).

61. Zhu, Z., Cheng, Y. \&Schwingenschlogl, U. Band inversion mechanism in topological insulators: A guideline for materials design. Physical Review B 85, 235401 (2012).

62. Perdew, J. P., Burke, K., \& Ernzerhof, M. Generalized Gradient Approximation Made Simple. Physical Review Letters 77, 3865 (1996).

63. Perdew, J. P., Burke, K., \& Ernzerhof, M. Generalized Gradient Approximation Made Simple. Physical Review Letters 78, 1396 (1997)

64. Troullier, N. \& Martins, J. L. Efficient pseudopotentials for plane-wave calculations. Physical Review B 43, 1993 (1991).

65. Feynman, R. P. Forces in Molecules. Physical Review 56, 340 (1939).

66. Hellmann, H. (1937). Einfuhrung in die Quantenchemie. Leipzig: Franz Deuticke. p. 285.

67. Vanderbilt, D. Soft self-consistent pseudopotentials in a generalized eigenvalue formalism. Physical Review B 41, 7892 (1990).

\section{Acknowledgements}

SMA is thankful to Dr. Ilya Ioffe (Moscow State University) and Parijat Sengupta (Purdue) for fruitful discussion and Purdue, MSE for financial support (Prof. Strachan). AAP is thankful to Deutsche Forschungsgemeinschaft (Project PO 1602/1-1) for financial support. PK and CGR acknowledges the Academy of Finland for funding. Authors greatly acknowledge the continuous support provided by Prof. Dunsch (IFW, Dresden) and Prof. Cuniberti (TU Dresden)

\section{Author contributions}

SMA suggested the idea. SMA and CGR performed theoretical analysis and numerical simulations, wrote the first draft of the manuscript and prepared the figures. $\mathrm{PK}, \mathrm{HC}$ and AAP contributed to the discussion of the results.

\section{Additional information}

Supplementary information accompanies this paper at http://www.nature.com/ scientificreports

Competing financial interests: The authors declare no competing financial interests.

License: This work is licensed under a Creative Common Attribution-NonCommercial-ShareAlike 3.0 Unported License. To view a copy of this license, visit http://creativecommons.org/licenses/by-nc-sa/3.0/

How to cite this article: Avdoshenko, S.M., Koskinen, P., Sevinçli, H., Popov, A.A. \& Rocha C.G. Topological Signatures in the Electronic Structure of Graphene Spirals.. Sci. Rep. 3, 1632; DOI:10.1038/srep01632 (2013) 